\title{
Study of dielectric properties of polyvinyl chloride (PVC) by the thermally stimulated current technique (TSC)
}

\author{
Mohamed OUAZENE
}

University of science and technology Houari Boumediene : Algiers, DZ-16000, and National Preparatory School to ingéniorat studies: Algiers, DZ-16012, Algiers, Algeria

Corresponding author: E-mail: ouaz_moh@yahoo.fr

Keywords: Polymers, Polyvinyl Chloride (PVC), Thermally Stimulated Depolarization Currents, Vogel Theory

\begin{abstract}
Polyvinyl chloride (PVC) is one of the polymers used in a plasticized state in the cable insulation to medium and high voltage. The studied material is polyvinyl chloride (PVC $4000 \mathrm{M}$ ) from the Algerian national oil company. Industrial PVC $4000 \mathrm{M}$ is in the form of white powder. The aim of this paper is to study the effect of temperature by the method of thermally stimulated depolarization currents. Furthermore, it is inferred from this work that the phenomenon of physical aging can have important consequences on the properties of the polymer. It leads to a more compact rearrangement of the material and a reconstruction or reinforcement of structural connections.
\end{abstract}

\section{Introduction}

THE insulating properties of the polymers are widely used in electrotechnics or in the insulation of high and medium voltage cables and many other uses [1]. In recent decades, the insulation of underground cables in power systems is made with polymeric materials because they have significant advantages technically and economically [2]. However, although the insulation with polymeric materials has important advantages, there are some disadvantages such as the effect of

heat that may have a detrimental effect on the properties of polymers [3]. Polyvinyl chloride (PVC) is one of the polymers used in a plasticized state in the cable insulation to medium and high voltage. The studied material is polyvinyl chloride (PVC $4000 \mathrm{M}$ ) from the Algerian national oil company. Industrial PVC 4000M is in the form of white powder. The test sample is a pastille of 1 $\mathrm{mm}$ thick and $1 \mathrm{~cm}$ in diameter.

\section{Experiment of tsc and maths A Experiment}

The sample is placed between two planar electrodes of circular shape within a cylindrical enclosure in which a vacuum has been carried out by a pump system. The two electrodes are held by two rigid rods, which are used at the same time to bring the electric current. A temperature sensor made in platinum (TN 2AS) positioned at the immediate vicinity of material allows to track the temperature variations over time. The probe is placed in a hole made in the ground electrode. The rise in temperature takes place by using a heating resistor placed near the sample.

The electrical diagram of the thermally stimulated method depolarization current is given by Fig 1. 


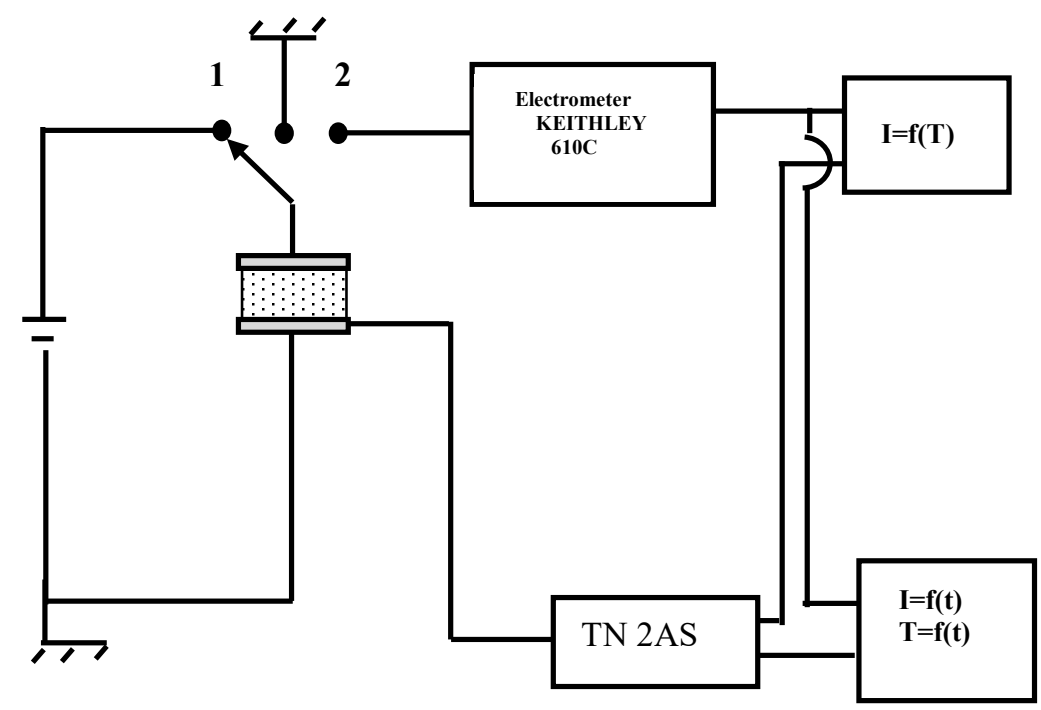

Fig 1. Circuit of measurement and recording

\section{$B \quad$ Relaxation in the polymers in the solid satete}

In polymers, we observe a relaxation of large amplitude, called the primary relaxation, and secondary relaxations occurring at temperatures below $\mathrm{Tg}$ [4]. The first said $\alpha$ relaxation associated with the glass transition originates from a large-scale rotation mechanism of the segments of the main chain [5]. These rotational movements, favored by the rubbery state wherein the polymer is $(\mathrm{T}>\mathrm{Tg})$, however, are held back by the friction movements due to interactions between mobile segments and the environment to which they belong. The local movements of the lateral dipoles of the main chain are also involved in the relaxation mechanism.

One of the molecular theories leading to relaxation time leads to Vogel equation:

$$
\tau=\tau_{0} \exp 1 / \alpha_{f}\left(T-T_{\infty}\right)
$$

where: $\boldsymbol{\alpha}_{\boldsymbol{f}}$ is the fraction of free volume, $\mathrm{T}$ is the temperature in $\mathrm{K}$ and $\boldsymbol{T}_{\infty}$ the temperature below which no rearrangement is possible. Representation of $\log \tau$ vs. 1 / T is linear.

\section{Résultats and discussions}

In a first step records the overall spectrum of the thermally stimulated depolarization current (TSC) in a PVC sample polarized at temperature $\mathrm{T}_{\mathrm{P}}=81^{\circ} \mathrm{C}$. This is illustrated by Fig. 2 . One notices a thermocourant peak at $\mathrm{T}=81^{\circ} \mathrm{C}$ preceded by a shoulder in the vicinity of $60^{\circ} \mathrm{C}$.

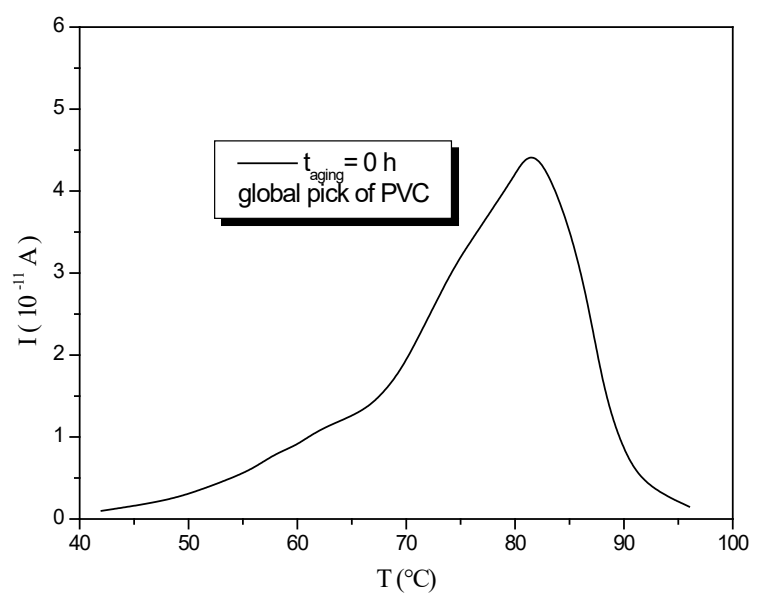

Fig. 2. Overall spectrum of TSDC of PVC at $T_{P}=81{ }^{\circ} \mathrm{C}$ 
The sample of PVC has undergone within the measurement cell stays of zero hour, 0.3 hour, 0.6 hour, one hour and two hours at temperature $\mathrm{Tg}=80^{\circ} \mathrm{C}$. It is then biased at this same temperature for two minutes with an electric field of $3.10^{5} \mathrm{~V} / \mathrm{m}$. The TSC technique is then applied and allows us to record the overall peaks corresponding to each time of aging as shown in Fig. 3.

One notices in this figure that the shape and position of the peaks do not seem to be affected by physical aging phenomenon. In the contrary, we note that the peak intensity decreases with the aging time. This result is similar to that found by Yianakopoulos G. et al. [2] by studying the aging P.V. C. For the isolated peak between $50^{\circ} \mathrm{C}$ and $60^{\circ} \mathrm{C}$, there is a temperature corresponding to each $\boldsymbol{T}_{\infty}$ aging time and which allows tracing $\log \tau$ vs. $1 /$ T as shown in Fig. 4.

Finally, note that the shape of the relaxation time spectrum does not change but there is movement of the latter towards the longest times. This leads us to say that the relaxation rate becomes more and more slow.

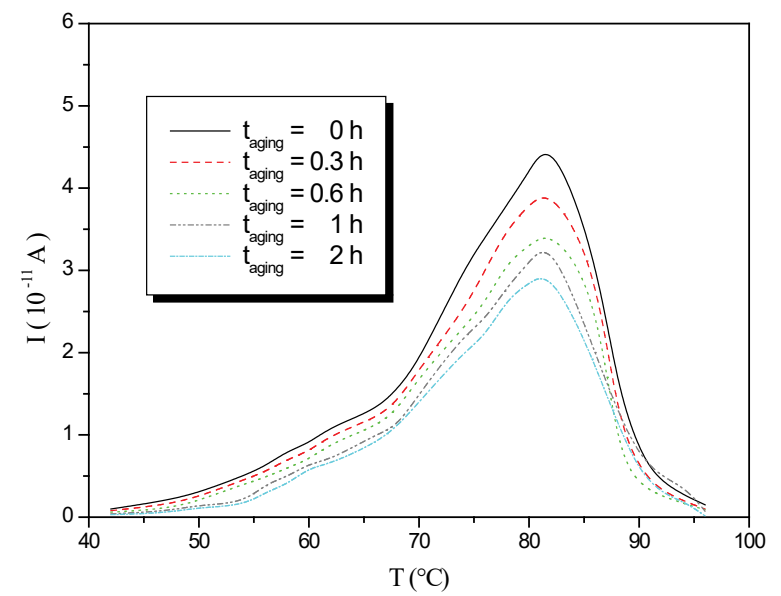

Fig.3. Overall peaks of aged PVC

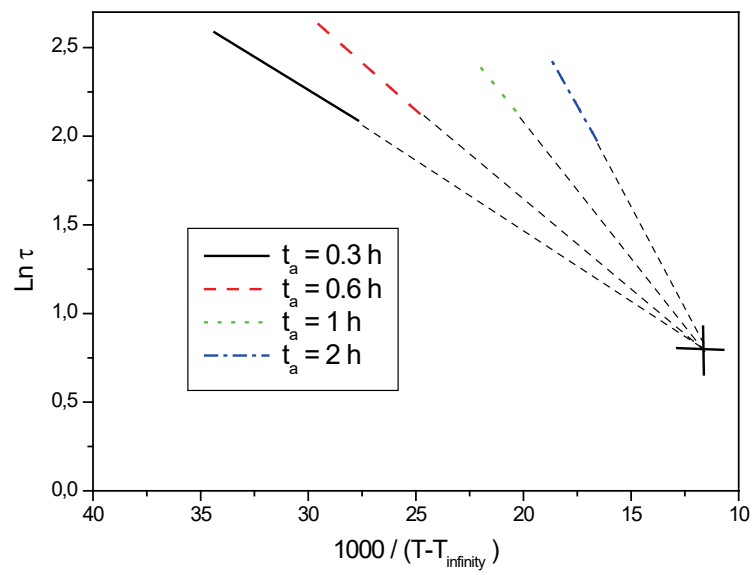

Fig.4. Vogel diagram of PVC aged at $T=60^{\circ} \mathrm{C}$

\section{Conclusion}

The technique of thermally stimulated depolarization currents has proven that it is well suited to highlight the physical aging due to the temperature of the studied polymer (PVC).

\section{References}

[1] A. Boudet, les polymères: leurs structures et leurs propriétés, extract of the book: Voyage au cœur de la matière plastique, CNRS-France, Editions, 2003, pp. 31-33.

[2] N. Doulache and M Bendaoud: "Study of the Influence of the Speed of Cooling on the $\alpha$ Relaxation in Polymers by the Thermostimulated Current Method," International Journal of Polymer 
Analysis and Characterization, Vol. 11(4), pp. 317-322, July 2006.

http://dx.doi.org/10.1080/10236660600754671

[3] S. Doulut, C. Bacharan, P. Demont, A. Bernès, and C. Lacabanne, "Physical aging and tacticity effects on the $\alpha$ relaxation mode of amorphous polymers by thermally stimulated techniques," Journal of Non-Crystalline Solids - Elsevier, Vol. 235-237, pp. 645-651, August 1998.

[4] C.Lacabanne and D. Chatain, "Depolarization thermocurrents in amorphous polymers," Journal of Polymer Science: Polymer Physics Edition, Vol. 11 (12),pp. 2315-2328, 11March 2003.

[5] Y. Yorozu, M. Hirano, K. Oka, and Y. Tagawa, "Electron spectroscopy studies on magnetooptical media and plastic substrate interface,” IEEE Transl. J. Magn. Japan, vol. 2, pp. 740-741, August 1987 [Digests 9th Annual Conf. Magnetics Japan, p. 301, 1982].

http://dx.doi.org/10.1109/TJMJ.1987.4549593 